\title{
Why Is Tetrahydrofuran a Good Solvent for Calcium Batteries? Insights From Ab Initio Molecular Dynamics Simulations
}

\author{
Shreyas Pathreeker, ${ }^{1}$ lan D. Hosein ${ }^{1 *}$ \\ 1. Syracuse University, Department of Biomedical and Chemical Engineering, \\ Syracuse, NY, 13244 \\ *Corresponding Author: idhosein@syr.edu
}

\section{Abstract}

Calcium batteries are rapidly emerging as a potential, future energy storage technology; however, their advancement relies heavily on understanding of the liquid electrolyte component in terms of stability and interactions with a calcium metal anode. Tetrahydrofuran, a cyclic ether, is an experimentally common and promising solvent for the preparation of stable and efficient calcium electrolytes. However, insights into the reasons why are lacking, which could unveil key principles to electrolyte design. In this report, we provide a theoretical study employing ab initio molecular dynamics (AIMD) simulations of the interactions of $\mathrm{Ca}$ metal with the cyclic ether tetrahydrofuran (THF). The results show that the electrochemical breakdown and decomposition of THF at the Ca surface is highly orientation- and surface-site dependent, thereby significantly reducing the likelihood of its instability in a randomly organized bulk solvent. Likewise, in bulk electrolytes, its likelihood for breakdown is further diminished, in preference for coordination $\mathrm{Ca}^{2+}$ to form solvated structure. Hence, the finding that molecules require such strict conditions for their decomposition is an important selection and design 
principle for any solvent to prepare suitable calcium electrolytes. These findings are critical to the advancement of the calcium batteries.

\section{Introduction}

Calcium batteries have emerged as a promising post-Li energy storage technology owing to its numerous benefits such as high energy density, competitive capacities and C-rates, vast and sustainable resource supply, low cost, and earth abundance. ${ }^{1}$ However, one of the challenges with their development is the discovery of suitable electrolytes. The strong reducing nature of calcium metal renders many solvents unstable, and likewise many electrolyte systems can decompose into products that passivate the metal surface, thereby shutting down Ca redox kinetics. ${ }^{2}$ And some stable electrolytes systems may only enable redox activity at elevated temperatures. ${ }^{3}$ Hence, the providing understanding of the electrochemistry of electrolytes is critical to the advancement of the calcium batteries. Electrolytes prepared with the well-known tetrahydrofuran (THF) has most recently emerged as an impressive solvent for the preparation of stable and efficient electrolytes. Several experimental reports have successfully demonstrated the use of THF (tetrahydrofuran) as a solvent in electrolytes for calcium (Ca) battery systems. ${ }^{4-11}$ However, a compelling argument as to the reason for its effectiveness as an electrolyte solvent has not been presented, other than the rationale of its equivalency to $\mathrm{Mg}$ electrochemistry. It is known that THF can react with $\mathrm{Ca}$ metal to form $\mathrm{CaH}_{2}$, which then forms a non-SEl-like protective layer on the $\mathrm{Ca}$ metal surface during reversible $\mathrm{Ca}$ plating and stripping. ${ }^{5,12}$ However, there is still ambiguity regarding the role of THF in the formation of calcium hydride $\left(\mathrm{CaH}_{2}\right)$. For instance, Shyamsunder and coworkers ${ }^{7}$ suggest that the formation of $\mathrm{CaH}_{2}$ in Wang et al's deposition of $\mathrm{Ca}$ in $\mathrm{THF}^{5}$ may be attributed to 
degradation of the salt (borohydride) anion, but Martinez-Cisneros and coworkers ${ }^{13}$ suggest that the formation of $\mathrm{CaH}_{2}$ is owing to decomposition of THF solvent. Furthermore, the chemical pathways for any possible THF decomposition remains to be explored and elucidated. Only a single report presents simulations of THF dynamics on calcium metal, ${ }^{14}$ yet at such elevated temperatures $(350 \mathrm{~K})$ as to possibly induce formation and decomposition chemical products that are unlikely at room temperature conditions, as a consequence of over-accelerated reaction kinetics. For example, we have shown experimentally ${ }^{12}$ using a calcium tetrafluoroborate $\left(\mathrm{Ca}(\mathrm{BF} 4)_{2}\right) /$ ethylene carbonate (EC):propylene carbonate (PC) electrolyte that the amount of calcium fluoride $\left(\mathrm{CaF}_{2}\right)$ formed is smaller at ambient temperature conditions than at elevated temperatures. ${ }^{3}$ Therefore, in-depth investigation of the interactions of THF solvent on $\mathrm{Ca}$ metal surfaces at room temperature condition is crucial. In this work, we computationally explore via ab initio molecular dynamics (AIMD) simulations the reductive breakdown mechanisms of THF at a Ca metal surface. We reveal stringent molecular orientation and location requirements of THF on the calcium metal surface to induce reductive breakdown such that in bulk, decomposition becomes probabilistically unlikely.

\section{Methods}

Density functional theory (DFT)-based structure optimization was carried out using the Vienna Ab-Initio Simulation Package (VASP) ${ }^{15} .1$ Atom positions were relaxed using the Conjugate Gradient algorithm with a convergence of $0.02 \mathrm{eV} / \mathrm{Ang}$. The DFT exchange-correlation energy was determined using the Generalized Gradient Approximation (GGA) Perdew-Burke-Ernzerhof (PBE) functional ${ }^{16}$. The planewave cutoff energy was $400 \mathrm{eV}$ and projector-augmented wave (PAW) pseudopotentials ${ }^{17}$ were 
used. First, a box of $10 \mathrm{THF}$ molecules was equilibrated to its target density of $0.88 \mathrm{~g} / \mathrm{mL}$, and then merged with an optimized $3 \times 2 \times 1$ calcium metal surface ([001]-terminated) to model the solvent-Ca-metal interface. Ab Initio Molecular Dynamics (AIMD) simulations up to $3000 \mathrm{fs}$ using the canonical (nVT) ensemble were performed with a timestep of $1 \mathrm{fs}$ on the optimized structures. The planewave cutoff energy was $400 \mathrm{eV}$, and energy convergence was $10^{-6} \mathrm{eV}$ using the Normal blocked Davidson algorithm. Gaussian smearing with a width of $0.05 \mathrm{eV}$ was used, as was a k-spacing of $1 / \AA$, i.e., the gammapoint, which led to a mesh size of $1 \times 1 \times 1$. This simulation was performed under $0 \mathrm{~V}$ conditions. Density of states (DOS) calculations were performed using the same energy cut-off, but with a k-spacing of $0.25 /$ Ang, and Tetrahedron smearing. All calculations and simulations were performed at a temperature of $298 \mathrm{~K}$ to represent room temperature conditions.

\section{$\underline{\text { Single-Molecule Studies }}$}

First, we investigated the effect of orientation and position of a single THF molecule on its potential breakdown characteristics and decomposition products. We isolated the solvent molecules in such a manner to explore non-competitive and exclusive solvent degradation characteristics in contact with the Ca metal surface, as reported previously in Li systems. ${ }^{18}$ In the context of electrochemistry of Ca metal, investigating solvent degradation products is key to understanding and designing interfaces that allow efficient and reversible deposition and dissolution of $\mathrm{Ca}$. Figure 1 shows a single THF solvent molecules placed over an equilibrated Ca metal surface in either of two orientations, i.e., planar and vertical, and either or two possible surface sites, i.e., the bridge position (between two $\mathrm{Ca}$ atoms) and top (on top of a $\mathrm{Ca}$ atom), thereby generating 4 distinct 
configurations. Snapshots after 3000 fs of AIMD simulation on the Ca metal surface are shown in Figures 1a)-d). In studies using THF as the solvent, it has been shown experimentally that $\mathrm{CaH}_{2}$ facilitates the reversible plating and stripping of $\mathrm{Ca}$. However, the origin of the $\mathrm{CaH}_{2}$ has been attributed to the salt $\mathrm{Ca}\left(\mathrm{BH}_{4}\right)_{2}$, and not necessarily the THF solvent. In our simulations, partial reduction of THF leading to hydrogen abstraction and consequent formation of $\mathrm{CaH}^{+}$and $\mathrm{CaC}_{4} \mathrm{H}_{7} \mathrm{O}^{+}$ion complexes were observed in the planar-bridge configuration (Figure 1a).
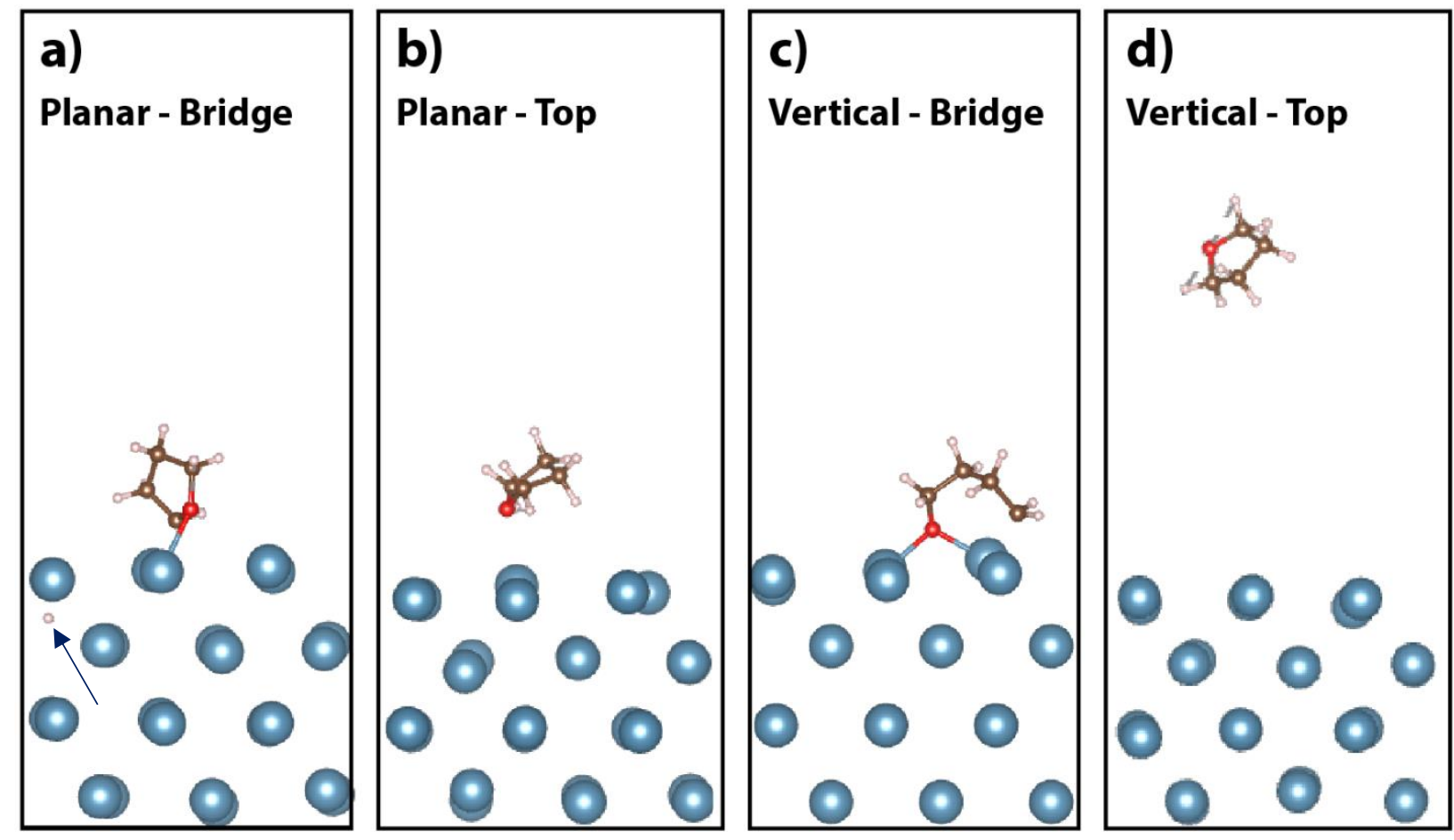

Figure 1. Final AIMD snapshots of single THF molecules initially placed in a) the planar-bridge position, $b$ ) planar-top, c) vertical-bridge, and d) vertical-top configurations. Blue is calcium, brown is calcium, white is hydrogen, and red is oxygen.

No hydrogen abstraction was found in the planar-top configuration, rather, the solvent was found to have detached and translated away from the metal surface (Figure 1b). Therefore, in the planar-bridge configuration, a second hydrogen abstraction, possibly from the salt molecule, could be required for the formation of $\mathrm{CaH}_{2}$, which may 
corroborate experimental findings that $\mathrm{Ca}\left(\mathrm{BH}_{4}\right)_{2}$ is involved in the formation of $\mathrm{CaH}_{2}$. In the vertical orientation, specifically in the vertical-bridge configuration (Figure 1c), ringopening of THF occurs. However, no further decomposition of the THF was observed following the ring-opening event. In the vertical-top configuration (Figure 1d), no ringopening was detected, but rather the THF molecule was found to detach and move away from the metal surface owing to lack of coordination with $\mathrm{Ca}$ atoms, similar to that seen in the planar-top configuration (Figure 1b). Overall, two types of breakdowns are observed from the evaluation of these 4 configurations: 1) hydrogen abstraction from THF in the planar-bridge position and 2) THF ring-opening in the vertical-bridge position. This contrasts with the well-known use of ethylene carbonate, for which decomposition is found for any orientation, apparently regardless of site location. Over the 4 configurations explored herein, these two breakdown events of hydrogen abstraction and ring-opening were both observed within the first 100 fs of the simulation. The total simulation time of 3000 fs employed in this work is sufficient to explore and reveal any THF reduction dynamics. Furthermore, previous reports have also employed a simulation time of 3000 fs. Recent simulation studies on the decomposition of EC on Ca metal also employ similar timescales of simulation. ${ }^{19}$

For the two configurations that lead to THF decomposition (Figures 1a) and 1c)), adsorption energies were calculated using the following equation,

$$
E_{\text {Adsorption }}=E_{\text {Slab:Solvent }}-E_{\text {Slab }}-E_{\text {Solvent }}
$$

wherein $E_{\text {Slab:Solvent }}$ is the energy of the solvent and the slab together, $\mathrm{E}_{\text {Slab }}$ is the energy of the Ca slab, and Esolvent is the energy of the solvent alone. The adsorption energy for the planar-bridge position was found to be $-0.418 \mathrm{eV} /$ atom and that for the vertical- 
bridge position was found to be $-0.42 \mathrm{eV} /$ atom, indicating that both positions are equally energetically favorable.

\section{Bond-Length Analysis}

Further analysis of the single solvent molecules shown in Figure 1 was conducted to understand the change in $\mathrm{C}-\mathrm{O}$ bond lengths over 500 fs of the AIMD simulation, as shown in correspond plots of Figure 2.
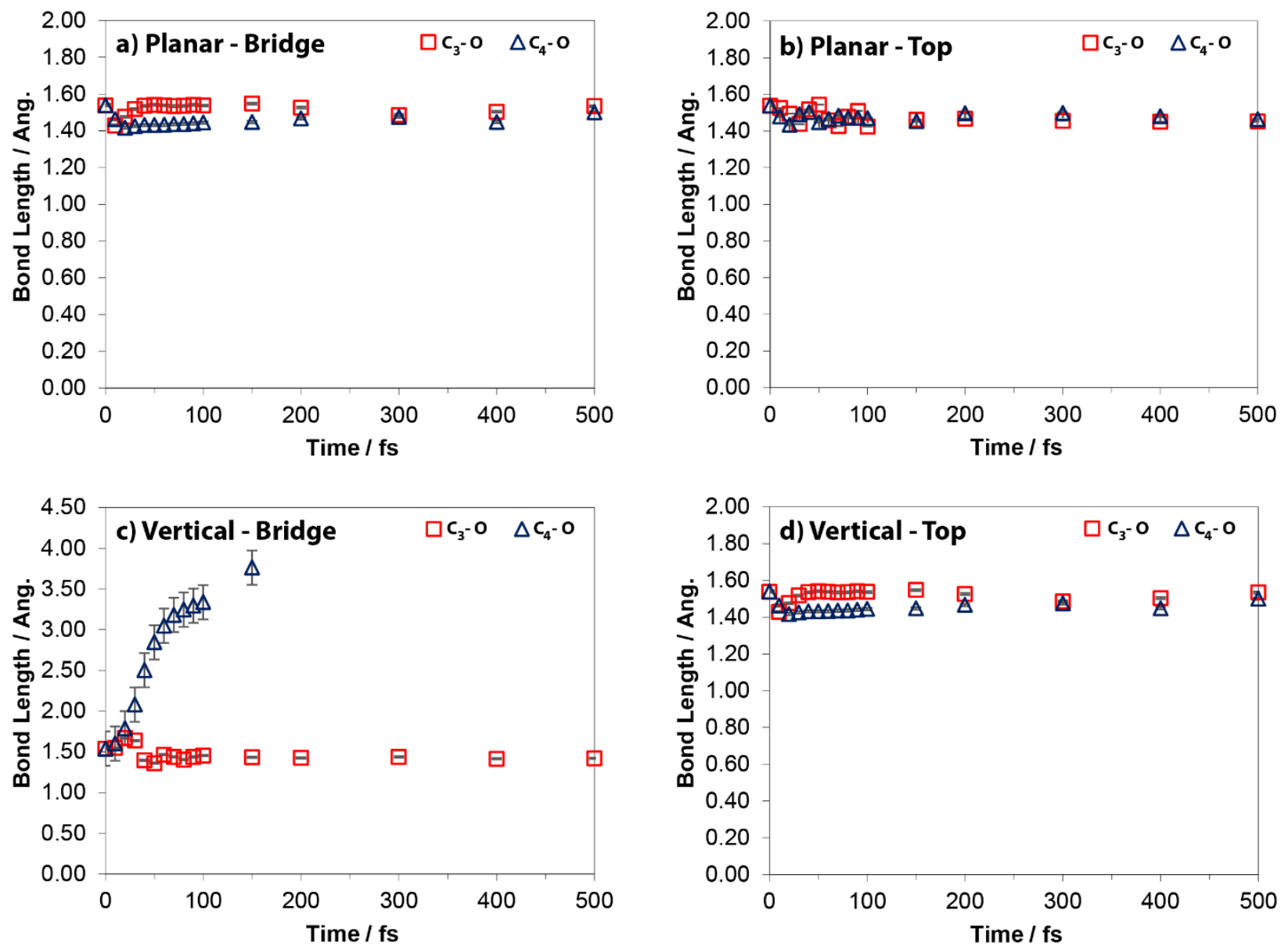

Figure 2. C-O bond lengths up to the first 500 fs in the simulation in different positions. a) vertical-bridge, b) planar-bridge, c) vertical-top, d) planar-top configurations.

In all (Figures 2a), 2b), 2d)) but the vertical-bridge configuration (Figure 2c), the $\mathrm{C}-\mathrm{O}$ bond lengths vary slightly initially, possibly to steric effects as can be seen from the AIMD 
snapshots, but then stabilize, thereafter remaining approximately the same as their initial bond-lengths. Whereas, in the vertical-bridge configuration (Figure 2c), one of the two C-O bonds stretches considerably and breaks at $\sim 150 \mathrm{fs}$, which then leads to ringopening of the THF molecule. This observation is in accordance with the ring-opening observed visually in the AIMD snapshots. It must be noted here that both the $\mathrm{C}-\mathrm{O}$ bonds present in the THF molecule are equally susceptible to cleavage by reduction. In this case, it appears that the THF C atom closer to the Ca surface owing to its proximity is subject to more electron transfer, and therefore, cleavage. Hence, from these simulations that, at the single-molecule level, THF is quite reductively stable when brought in contact with Ca metal, in that, it does not breakdown completely to form any distinct products.

The primary mechanism of solvent breakdown at $0 \mathrm{~V}$ conditions is charge (electron) transfer from the metal surface to the solvent molecule. To this end, we conducted Bader charge analysis ${ }^{20-22}$ on the simulation boxes shown in Figure 1. Since solvent breakdown herein was only observed in two configurations (planar-bridge and vertical-bridge), our Bader charge analysis was limited to these two configurations. Charges on the $\mathrm{C}$ atoms connected to the terminal $\mathrm{O}$ atom were averaged together, resulting in a single charge value for $\mathrm{C}$, and another for $\mathrm{O}$. In the planar-bridge configuration, charge transfer between $\mathrm{Ca}$ metal and the $\mathrm{C}$ and $\mathrm{O}$ atoms of the THF molecule was found to be $-0.35 e$, and $0 e$, respectively, translating to a $\sim 0.35 e$ loss from the THF molecule through the $\mathrm{C}$ atoms, which could be due to a combination of hydrogen abstraction and steric stabilization. In the vertical-bridge configuration, charge transfer between the $\mathrm{Ca}$ metal and the $\mathrm{C}$ and $\mathrm{O}$ atoms of the THF molecule was found to be $+0.15 e$, and $+0.8 e$, respectively. This charge transfer corresponds to a $\sim 1 e$ gain by the 
THF molecule, which points towards a $1 e$ reduction process. Interestingly, maximum charge transfer occurs through the oxygen atom. Similar findings have been reported with EC on Ca metal. ${ }^{19}$ In the planar-top and vertical-top configurations, charge transfer was found to be negligible (see Figure S1, Supporting Information).

To explore the decomposition pathways associated with these two configurations, transition state searches were conducted in the vertical-bridge and planar-bridge positions to reveal the energy profile and decomposition pathway for THF. A small saddle point was found for the planar-bridge configuration, which coincided with the hydrogen abstraction event (see Figure S2, Supporting Information). However, no distinct transition states were found for the vertical-bridge configuration (see Figure S3, Supporting Information) owing to rapid breakdown of the THF followed by steric stabilization. From the single-molecule studies shown so far, THF is susceptible to hydrogen abstraction and/or ring-opening due to electron transfer from the Ca metal surface. However, importantly, these pathways for breakdown are highly dependent upon THF orientation and location over the Ca surface.

\section{Multiple THF Molecules to Emulate Bulk Solvent Phase}

The single-molecule configurations revealed the fundamental decomposition processes at the single-molecule scale, namely hydrogen abstraction and ring-opening. To capture a more realistic picture of THF breakdown dynamics and processes in the bulk solvent against a Ca metal surface, we examined several THF molecules (pure solvent, without salt) packed randomly (placement and orientation) over the Ca metal surface to emulate the bulk solvent. Figure 3 shows AIMD snapshots of 10 THF molecules obtained over a simulation time of $3000 \mathrm{fs}$ ( $3 \mathrm{ps}$ ). 


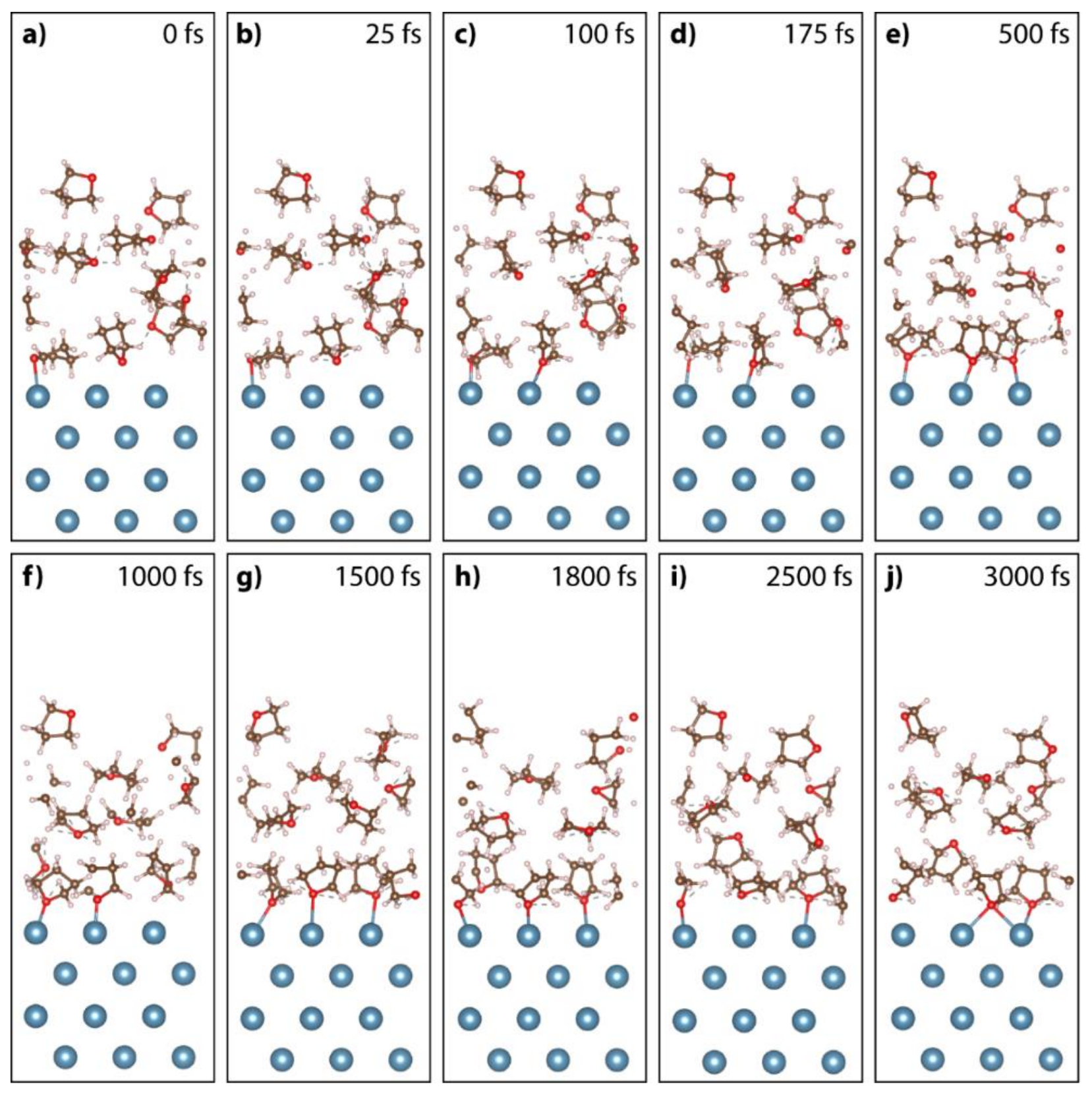

Figure 3. AIMD snapshots of $10 \mathrm{THF}$ molecules without salt. Blue is calcium, brown is calcium, white is hydrogen, and red is oxygen.

It was observed in such a bulk, randomly orientation THF molecule system that all molecules remained stable, and neither of the two breakdown mechanisms shown earlier are observed. While several atoms from the solvent molecules at the Ca surface do coordinate with the $\mathrm{Ca}$ atoms, they remain stable thereafter with not breakdown event 
over the rest of the simulation time, in contrast with the individual configurations wherein both these breakdown processes are observed (Figure 1). We confirmed the coordination and interaction of the THF molecules with the surface $\mathrm{Ca}$ atoms with density of states (DOS) calculations after AIMD simulation, wherein hybridization of the surface Ca atoms with oxygen atoms from the THF molecule was evident (representative data is shown in Figure S4, Supporting Information).

Hence, our observations further corroborate that the specific events of hydrogen abstraction and ring-opening are highly orientation-dependent owing to the specific pathways of electron transfer through an individual THF molecule. Despite the time for coordination and interactions with the Ca surface, whereby it would be possible for the THF molecules to assume the orientations and positions for which breakdown could be induced, not such decomposition is observed. Hence, the lack of any significant decomposition of THF in a pseudo-bulk state shown here, owing to the highly specific locations and orientations at which decomposition can occur, indicates that overall THF is a stable solvent at $0 \mathrm{~V}$ conditions, affirming experimental observations of its stability. This observation for THF is unlike ethylene carbonate, which is known to form distinct decomposition products as per simulations reported by Young and coworkers. ${ }^{19}$ This stability of THF against the Ca metal surface is likely the reason behind the popular use of THF as a solvent in experimental studies of $\mathrm{Ca}$ metal redox activity.

\section{Multiple THF and Salt Molecules to Emulate Bulk Electrolyte}

We further investigated the potential breakdown of THF in the presence of $\mathrm{Ca}\left(\mathrm{BF}_{4}\right)_{2}, \mathrm{Ca}(\mathrm{TFSI})_{2}$, and $\mathrm{Ca}\left(\mathrm{ClO}_{4}\right)_{2}$, to more accurately emulated bulk electrolytes (solvent + salt). Final snapshots from these simulations are shown in Figure 4. No THF 
decomposition was observed in the presence of $\mathrm{Ca}\left(\mathrm{BF}_{4}\right)_{2}$ and $\mathrm{Ca}(\mathrm{TFSI})_{2}$, in accordance with the results obtained in pure solvent (Figure 3), thus reinforcing the reductive stability of THF solvent.
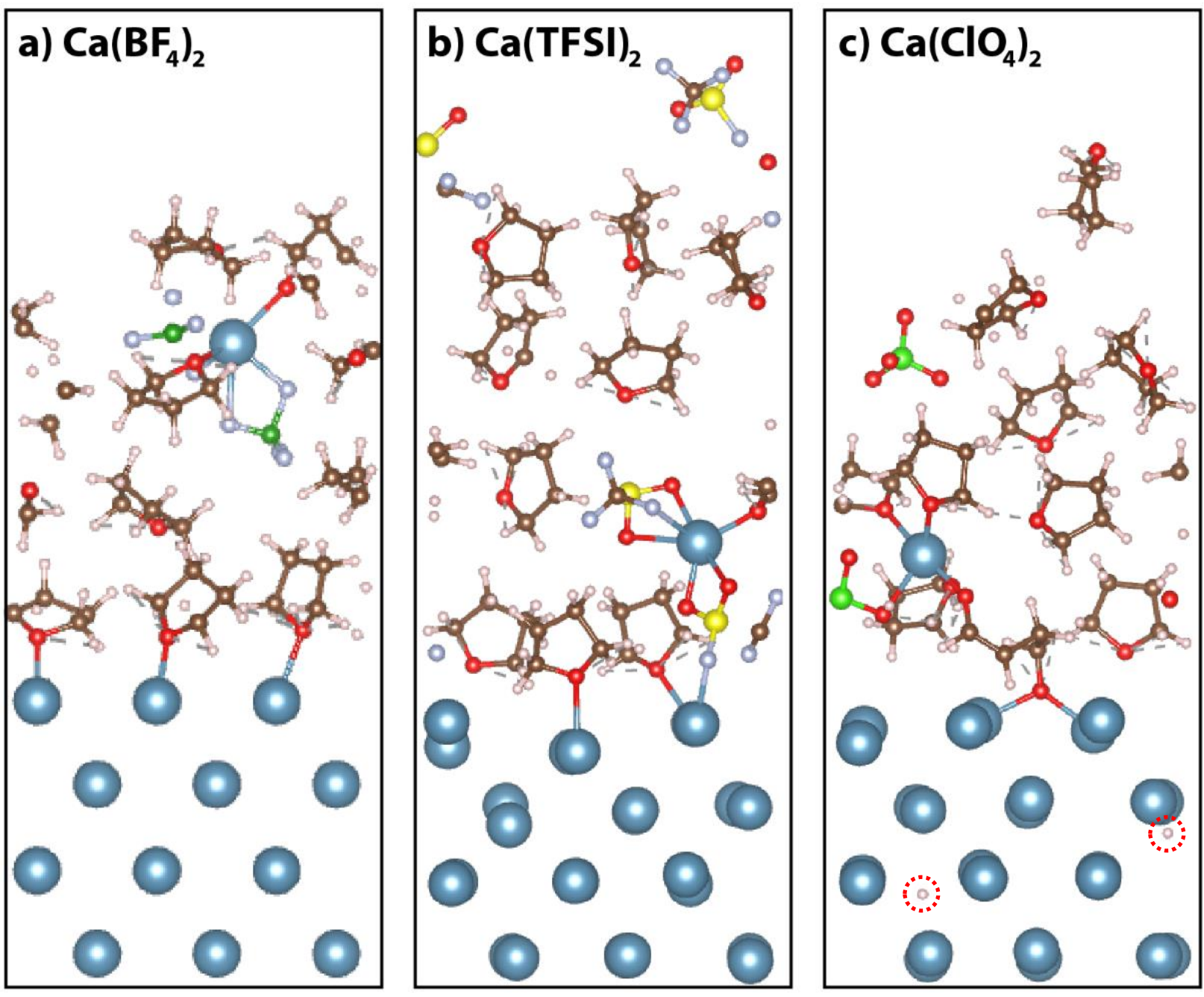

Figure 4. AIMD snapshots of $10 \mathrm{THF}$ molecules with a) $\mathrm{Ca}\left(\mathrm{BF}_{4}\right)_{2}$ (green is boron, light blue is fluorine), b) $\mathrm{Ca}(\mathrm{TFSI})_{2}$ (Yellow is sulfur, light blue atoms connected to yellow atoms are nitrogen, light blue atoms connected to brown atoms are fluorine), and c) $\mathrm{Ca}\left(\mathrm{ClO}_{4}\right)_{2}$ (green is chlorine). Blue is calcium, brown is calcium, white is hydrogen, and red is oxygen.

Notably, while salt anions were in proximity to the Ca metal surface, no decomposition of the anions was observed. However, hydrogen abstraction was indeed observed in the presence of $\mathrm{Ca}\left(\mathrm{ClO}_{4}\right)_{2}$, which appears to be solely from THF and due to the orientation 
of the THF molecule. In theory, this implies the formation of $\mathrm{CaH}_{2}$, the origin of which in experimental studies has been left open to interpretation, as mentioned previously. For instance, in their experimental investigation of $\mathrm{Ca}$ metal plating and stripping in $\mathrm{Ca}\left(\mathrm{BH}_{4}\right)_{2}-$ THF electrolyte, Wang and Bruce ${ }^{5}$ found $\mathrm{CaH}_{2}$ in their deposits, whose origin was attributed to degradation of the $\mathrm{Ca}\left(\mathrm{BH}_{4}\right)_{2}$ salt. Gewirth and coworkers ${ }^{11}$ also reported from their experimental studies that hydrogen abstraction was necessary to form $\mathrm{CaH}_{2}$ in the presence of $\mathrm{Ca}\left(\mathrm{BH}_{4}\right)_{2}$ salt. However, no evidence of THF decomposition was found after GC-MS characterization of a piece of Ca metal dipped into THF solvent. It was suggested by the authors that THF may still decompose to form $\mathrm{CaH}_{2}$, still leaving the topic of THF stability ambiguous. Although these authors did not find any hydride products in their deposits, $\mathrm{C}_{4} \mathrm{H}_{6} \mathrm{O}$ was detected. When compared to other similar electrolyte systems, in an $\mathrm{EC} / \mathrm{Ca}\left(\mathrm{ClO}_{4}\right)_{2}$ system investigated by Young and coworkers, ${ }^{19}$ the presence of $\mathrm{Ca}\left(\mathrm{ClO}_{4}\right)_{2}$ molecules was found to accelerate the breakdown of $\mathrm{EC}$ on a Ca metal surface at temperatures $\geq 350 \mathrm{~K}$. Additionally, the $\mathrm{ClO}_{4}^{-}$anion was also found to further decompose into other products. However, it appears that the effect of salt Ca cationsolvent coordination was not considered by the authors, and these decomposition products were reported at elevated temperatures.

\section{Coordination Environment in the Electrolyte}

While no decomposition of the salt (anion) molecules was observed for any salt explored herein, the effect of electrolyte solvation dynamics on solvent degradation was assessed. The objective of this computational study is to investigate the possible decomposition products of THF and salt molecules on a Ca metal surface. While the possible decomposition product from THF solvent is $\mathrm{CaH}_{2}$ owing to Hydrogen abstraction, 
the alteration of THF degradation dynamics in the presence of salt molecules is presented hereon. Figure 5a) shows the number of THF molecules coordinating with the surface Ca metal atoms. The number of solvent molecules coordinating with the Ca metal surface at the end of the simulation follows the order no salt $>\mathrm{Ca}\left(\mathrm{BF}_{4}\right)_{2}=\mathrm{Ca}(\mathrm{TFSI})_{2}>\mathrm{Ca}\left(\mathrm{ClO}_{4}\right)_{2}$, indicating that overall, more solvent molecules interact and coordinate with the Ca metal surface in the presence of more strongly coordinating $\mathrm{Ca}$ salt like $\mathrm{Ca}\left(\mathrm{BF}_{4}\right)_{2}$. The number of solvent and/or salt molecules available for coordination with the surface Ca metal atoms depends upon the solvation characteristics of the salt $\mathrm{Ca}$ cation. It is well known that local Ca cation coordination in the solvent plays an important role in reversible plating and stripping of Ca metal. ${ }^{23-24}$ Our AIMD simulations allow the visualization of solvated structures in the electrolyte.

a) No. of THF Molecules on Surface Ca Atoms

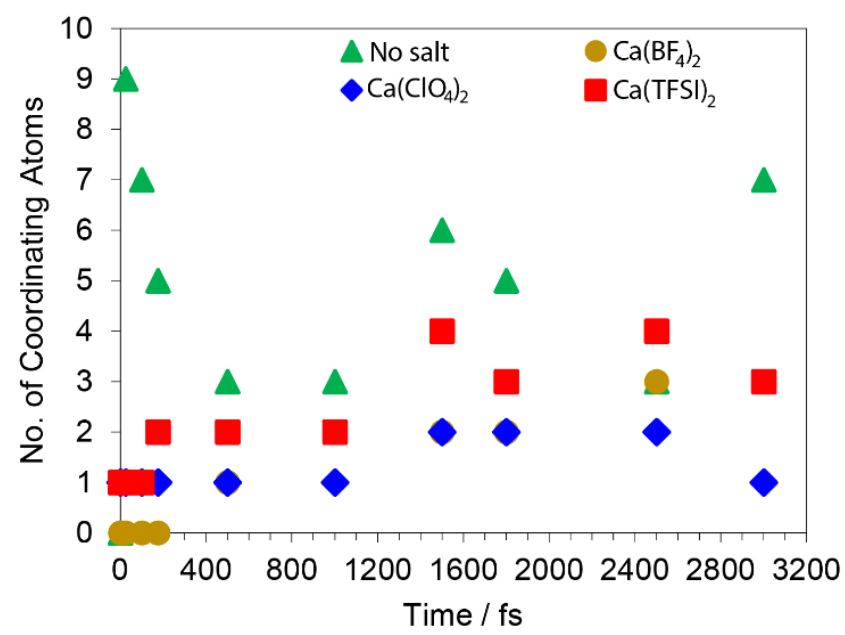

b) No. of Salt Anions and THF w/ Salt Ca Cation

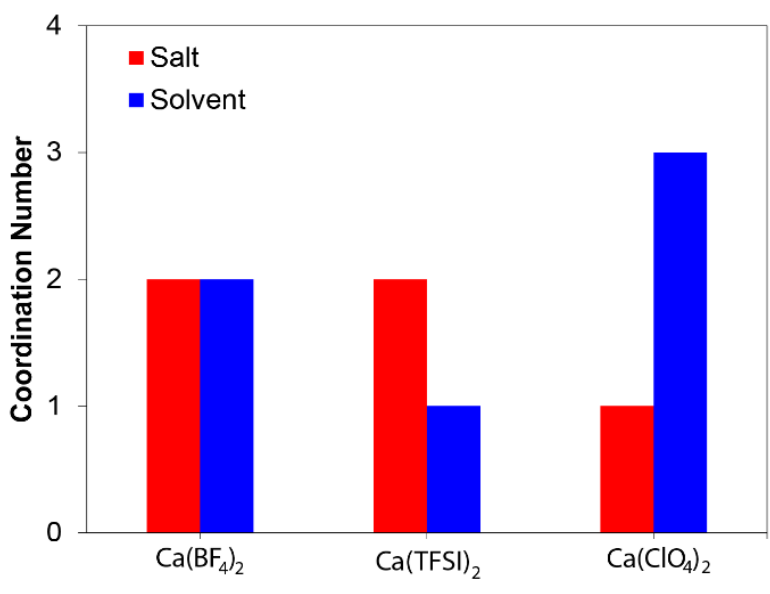

Figure 5. Plots of a) the number of solvent molecules coordinating with the surface $\mathrm{Ca}$ atoms in the presence of different salts, and b) the number of salt and solvent molecules coordinating with the salt $\mathrm{Ca}$ cation in solution.

Figure 5b) shows the number of salt anion and THF atoms coordinating with the salt $\mathrm{Ca}$ cation in the electrolyte. The solvated structures consist of both solvent and salt anion molecules (mixed ion-pairs), which is in contrast to the more specific SSIPs and CIPS 
reported elsewhere. ${ }^{25}$ Based on our AIMD simulations in the presence of salt molecules (Figure 4) at the end of the 3000 fs simulation times, we found salt Ca cation-THF solvent coordination to be highest for $\mathrm{Ca}\left(\mathrm{ClO}_{4}\right)_{2}$ ( 3 solvent atoms), followed by $\mathrm{Ca}\left(\mathrm{BF}_{4}\right)_{2}$ (2 solvent atoms), whereas only 1 solvent atom was found to coordinate with the electrolyte $\mathrm{Ca}$ atom for $\mathrm{Ca}(\mathrm{TFSI})_{2}$. On the other hand, coordination of salt anions with the salt $\mathrm{Ca}$ cation in the electrolyte was found to be highest for $\mathrm{Ca}(\mathrm{TFSI})_{2}$ ( 3 atoms) and $\mathrm{Ca}\left(\mathrm{BF}_{4}\right)_{2}(3$ atoms) followed by $\mathrm{Ca}\left(\mathrm{ClO}_{4}\right)_{2}$ (1 atom). These numbers are summarized in Table 1. Recalling the 2 hydrogen abstractions seen in Figure 4c), our results indicate that a strongly THF-coordinated $\mathrm{Ca}$ ion (see $\mathrm{Ca}\left(\mathrm{ClO}_{4}\right)_{2}$ ) could lead to selective decomposition of the THF molecule closest to the Ca metal surface. Interestingly, in this case, the decomposed solvent molecule was also coordinated with the salt $\mathrm{Ca}$ cation. One plausible explanation for this observation is the destabilization of the coordinating solvent in the case of strongly THF solvated salt Ca cations. Hahn and coworkers ${ }^{23}$ have suggested such behavior in their work using THF, and reported that strong solvent coordination can hinder the deposition of $\mathrm{Ca}$ metal. Therefore, when salt $\mathrm{Ca}$ cationsolvent coordination (or SSIP) is high, such as with $\mathrm{Ca}\left(\mathrm{ClO}_{4}\right)_{2}$, $\mathrm{Ca}$ ion desolvation from the bulk electrolyte and subsequent plating can be energetically unfavorable. In terms of the decomposition products, Driscoll and coworkers ${ }^{24}$ propose that in electrolyte systems consisting of THF solvent, the decomposition products may be anion-derived, as more anions can interact with Ca metal surface. However, this observation is not evident in our simulations. In other words, strong coordination of THF solvent to the Ca metal surface helps mitigate salt decomposition. 
Table 1: Summary of the number of salt and solvent molecules coordinating with the salt Ca cation

\begin{tabular}{ccc}
\hline Salt & $\begin{array}{c}\text { No. of } \\
\text { Solvents }\end{array}$ & $\begin{array}{c}\text { No. of Salt } \\
\text { Anions }\end{array}$ \\
\hline $\mathrm{Ca}(\mathrm{BF})_{2}$ & 2 & 3 \\
$\mathrm{Ca}(\mathrm{TFSI})_{2}$ & 1 & 3 \\
$\mathrm{Ca}\left(\mathrm{ClO}_{4}\right)_{2}$ & 3 & 1 \\
\hline
\end{tabular}

Overall, our results suggest that the presence of salt molecules in the electrolyte does not significantly alter the reductive stability of the THF solvent at the Ca metal surface. The stability of THF solvent has been reported previously both in the $\mathrm{Ca}$ and the $\mathrm{Mg}$ literature by Hahn and coworkers, ${ }^{23}$ and Bucur and coworkers, ${ }^{26}$ respectively. Our simulations confirm that THF can be the sole source of hydrogen to enable formation of $\mathrm{CaH}_{2}$ at room temperature, but only in highly selective orientations and positions. The possibility of $\mathrm{CaH}_{2}$ formation solely from the THF solvent molecule can be a useful prediction, since $\mathrm{CaH}_{2}$ is known to be an effective decomposition product comprising a $\mathrm{Ca}^{2+}$-permeable solid electrolyte interface (SEI) suitable for reversible plating and stripping of Ca metal. While THF appears to be a universally stable solvent, there could be certain highly selective orientations (such as planar-bridge) by which it can contribute to the production of $\mathrm{CaH}_{2}$. 


\section{Conclusion}

While several factors such as solubility, desolvation energy, ion-pairing, and iondiffusivity, are important in the design of battery electrolytes, the aim of this work was specifically to assess the reductive stability of THF solvent against a Ca metal surface. From this standpoint, our results clearly reveal that THF is a stable electrolyte solvent for $\mathrm{Ca}$ batteries at $0 \mathrm{~V}$ conditions. Namely, the only possible decomposition product from THF is $\mathrm{CaH}_{2}$, which is furthermore highly orientation- and location- dependent. The degradation mechanism of commonly used Ca salts has already been reported in the literature, which we acknowledge can change the overall mechanisms of Ca transport, plating, stripping, and the formation of the SEI layer. In this work, it appears that there are no salt decomposition products at room temperature. Our findings indicate the principle that solvent molecules that have very orientation- and site-specific triggers for decomposition pathways on $\mathrm{Ca}$ metal can be thereby sufficiently stable in bulk electrolytes. Differences in the Ca solvation shells for each salt as well as their effect on electrolyte degradation are subjects of current study and are being reported elsewhere.

\section{Supporting Information}

Bader charges as functions of simulation time (Figure S1); Transition state search for the planar-bridge configuration (Figure S2); Transition state search for the vertical-bridge configuration (Figure S3); Density of states (DOS) (Figure S4) 


\section{Acknowledgments}

The authors gratefully acknowledge support from the National Science Foundation under grant number CMMI-1751621, the College of Engineering and Computer Science at Syracuse University, and the Syracuse Center of Excellence.

\section{$\underline{\text { References }}$}

1. Hosein, I. D., The Promise of Calcium Batteries: Open Perspectives and Fair Comparisons. ACS Energy Lett. 2021, 6, 1560-1565.

2. Aurbach, D.; Skaletsky, R.; Gofer, Y., The Electrochemical-Behavior of Calcium Electrodes in a Few Organic Electrolytes. J. Electrochem. Soc. 1991, 138, 3536-3545.

3. Ponrouch, A.; Frontera, C.; Barde, F.; Palacin, M. R., Towards a Calcium-Based Rechargeable Battery. Nat. Mater. 2016, 15, 169-173.

4. Aurbach, D.; Skaletsky, R.; Gofer, Y., The Electrochemical Behavior of Calcium Electrodes in a Few Organic Electrolytes. Journal of The Electrochemical Society 1991, 138, 3536-3545.

5. Wang, D.; Gao, X.; Chen, Y.; Jin, L.; Kuss, C.; Bruce, P. G., Plating and Stripping Calcium in an Organic Electrolyte. Nat Mater 2018, 17, 16-20.

6. Li, Z.; Fuhr, O.; Fichtner, M.; Zhao-Karger, Z., Towards Stable and Efficient Electrolytes

for Room-Temperature Rechargeable Calcium Batteries. Energy \& Environmental Science 2019, 12, 3496-3501.

7. Shyamsunder, A.; Blanc, L. E.; Assoud, A.; Nazar, L. F., Reversible Calcium Plating and Stripping at Room Temperature Using a Borate Salt. ACS Energy Lett. 2019, 4, 2271 2276.

8. Jie, Y.; Tan, Y.; Li, L.; Han, Y.; Xu, S.; Zhao, Z.; Cao, R.; Ren, X.; Huang, F.; Lei, Z.; Tao, G.; Zhang, G.; Jiao, S., Electrolyte Solvation Manipulation Enables Unprecedented Room-Temperature Calcium-Metal Batteries. Angew Chem Int Ed Engl 2020, 59, 12689 12693.

9. Melemed, A. M.; Gallant, B. M., Electrochemical Signatures of Interface-Dominated Behavior in the Testing of Calcium Foil Anodes. Journal of The Electrochemical Society 2020, 167, 140543.

10. Kisu, K.; Kim, S.; Shinohara, T.; Zhao, K.; Züttel, A.; Orimo, S.-i., Monocarborane Cluster as a Stable Fluorine-Free Calcium Battery Electrolyte. Scientific Reports 2021, $11,7563$.

11. Ta, K.; Zhang, R.; Shin, M.; Rooney, R. T.; Neumann, E. K.; Gewirth, A. A., Understanding $\mathrm{Ca}$ Electrodeposition and Speciation Processes in Nonaqueous Electrolytes for Next-Generation Ca-Ion Batteries. ACS Appl Mater Interfaces 2019, 11, 21536-21542.

12. Biria, S.; Pathreeker, S.; Li, H.; Hosein, I. D., Plating and Stripping of Calcium in an Alkyl Carbonate Electrolyte at Room Temperature. ACS Applied Energy Materials 2019, 2, 7738-7743. 
13. Martinez-Cisneros, C. S.; Fernandez, A.; Antonelli, C.; Levenfeld, B.; Varez, A.; Vezzù, K.; Di Noto, V.; Sanchez, J. Y., Opening the Door to Liquid-Free Polymer Electrolytes for Calcium Batteries. Electrochim. Acta 2020, 353, 136525.

14. Liepinya, D.; Smeu, M., A Computational Comparison of Ether and Ester Electrolyte Stability on a Ca Metal Anode.

15. Kresse, G.; Furthmüller, J., Efficient Iterative Schemes for Ab Initio Total-Energy Calculations Using a Plane-Wave Basis Set. Phys Rev B 1996, 54, 11169-11186.

16. Perdew, J. P.; Burke, K.; Ernzerhof, M., Generalized Gradient Approximation Made Simple. Phys. Rev. Lett. 1996, 77, 3865-3868.

17. Blöchl, P. E., Projector Augmented-Wave Method. Phys Rev B 1994, 50, 1795317979.

18. Ebadi, M.; Brandell, D.; Araujo, C. M., Electrolyte Decomposition on Li-Metal Surfaces from First-Principles Theory. J Chem Phys 2016, 145, 204701.

19. Young, J.; Smeu, M., Ethylene Carbonate-Based Electrolyte Decomposition and Solid-Electrolyte Interphase Formation on Ca Metal Anodes. J Phys Chem Lett 2018, 9, 3295-3300.

20. Bader, R. F. W., Atoms in Molecules. Accounts of Chemical Research 1985, 18, 915.

21. Henkelman, G.; Arnaldsson, A.; Jónsson, H., A Fast and Robust Algorithm for Bader Decomposition of Charge Density. Computational Materials Science 2006, 36, 354-360. 22. Sanville, E.; Kenny, S. D.; Smith, R.; Henkelman, G., Improved Grid-Based Algorithm for Bader Charge Allocation. J Comput Chem 2007, 28, 899-908.

23. Hahn, N. T.; Driscoll, D. M.; Yu, Z.; Sterbinsky, G. E.; Cheng, L.; Balasubramanian, M.; Zavadil, K. R., Influence of Ether Solvent and Anion Coordination on Electrochemical Behavior in Calcium Battery Electrolytes. ACS Appl. Energy Mater. 2020, 3, 8437-8447. 24. Driscoll, D. M.; Dandu, N. K.; Hahn, N. T.; Seguin, T. J.; Persson, K. A.; Zavadil, K. R.; Curtiss, L. A.; Balasubramanian, M., Rationalizing Calcium Electrodeposition Behavior by Quantifying Ethereal Solvation Effects on Ca2+ Coordination in WellDissociated Electrolytes. Journal of The Electrochemical Society 2020, 167, 160512.

25. Agarwal, G.; Howard, J. D.; Prabhakaran, V.; Johnson, G. E.; Murugesan, V.; Mueller, K. T.; Curtiss, L. A.; Assary, R. S., Insights into Spontaneous Solid Electrolyte Interphase Formation at Magnesium Metal Anode Surface from Ab Initio Molecular Dynamics Simulations. ACS Applied Materials \& Interfaces 2021, 13, 38816-38825.

26. Muldoon, J.; Bucur, C. B.; Gregory, T., Quest for Nonaqueous Multivalent Secondary Batteries: Magnesium and Beyond. Chem. Rev. 2014, 114, 11683-11720. 
FOR TABLE OF CONTENTS USE ONLY

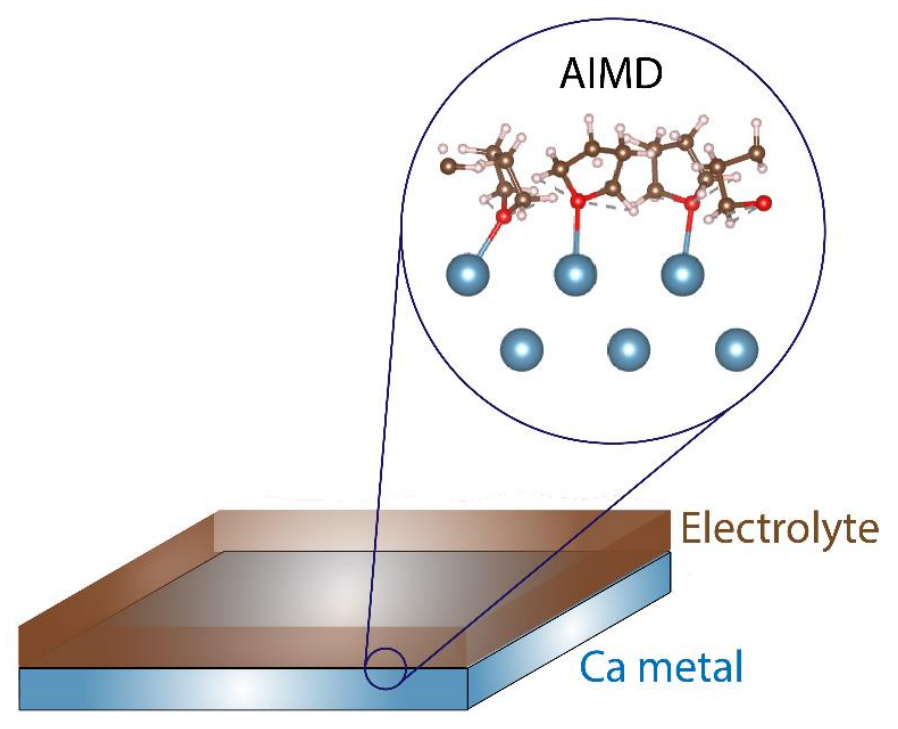

